\title{
A Compact Quadrature Hybrid MMIC Using CMOS Active Inductors
}

\author{
Hsieh-Hung Hsieh, Student Member, IEEE, Yu-Te Liao, Student Member, IEEE, and Liang-Hung Lu, Member, IEEE
}

\begin{abstract}
A miniaturization technique for the quadrature hybrid is proposed for monolithic-microwave integrated-circuit applications. By using active inductors for the circuit implementation, a significant area reduction can be achieved due to the absence of distributed components and spiral inductors. Using a $0.18-\mu \mathrm{m}$ bulk CMOS process, a 4.2-GHz quadrature hybrid is implemented for demonstration. The chip size measures $0.72 \times 0.64 \mathrm{~mm}^{2}$ where the hybrid circuit only occupies active area of $0.4 \times 0.2 \mathrm{~mm}^{2}$. With the enhanced $Q$ factor provided by the active inductors, the fabricated circuit has an insertion loss less than $0.4 \mathrm{~dB}$ for -3-dB coupling while maintaining excellent return losses and port isolation in the vicinity of the center frequency. Owing to the reconfigurable capability provided by the CMOS active inductors, the center frequency of the quadrature hybrid can be varied from 3.6 to $4.7 \mathrm{GHz}$ by adjusting the bias currents, exhibiting a tuning range of $26 \%$ at $C$-band.
\end{abstract}

Index Terms-CMOS active inductors, insertion loss, lumped components, miniaturization, monolithic microwave integrated circuit (MMIC), quadrature hybrid.

\section{INTRODUCTION}

$\mathbf{T}$ HE quadrature hybrid is a four-port network, which behaves as a directional coupler. Within the operating bandwidth, equal power dividing with a $90^{\circ}$ phase difference is provided at the outputs of the through and the coupled port, while zero signal coupling is exhibited at the isolation port. Since the quadrature hybrid is suitable for balanced circuit operations, it has been widely used in microwave systems for decades. In a planar process technology, the circuit is typically realized by the branch-line architecture. Owing to the use of four quarter-wavelength transmission line components in conventional circuit implementations, the size of the quadrature hybrid is prohibitively large, making it impractical for monolithic-microwave integrated-circuit (MMIC) applications, especially at frequencies below $10 \mathrm{GHz}$.

In order to reduce the chip area, techniques have been proposed for the quadrature hybrids by using multilayer configurations [1], [2], 3-D technologies [3], and lumped [4]-[7] or lumped-distributed equivalence [8]. However, due to the lack of high- $Q$ on-chip passive components, most of the miniaturized circuits suffer from high insertion losses, large phase error,

Manuscript received December 1, 2006; revised February 8, 2007. This work was supported in part by the National Science Council under Grant 94-2220E-002-026 and Grant 94-2220-E-002-009.

H.-H. Hsieh and L.-H. Lu are with the Department of Electrical Engineering and Graduate Institute of Electronics Engineering, National Taiwan University, Taipei, Taiwan 10617, R.O.C. (e-mail: lhlu@cc.ee.ntu.edu.tw).

Y.-T. Liao was with the Graduate Institute of Electronics Engineering, National Taiwan University, Taipei, Taiwan, 10617, R.O.C. He is now with the Department of Electrical Engineering, University of Washington, Seattle, WA 98195 USA.

Digital Object Identifier 10.1109/TMTT.2007.896815 and unequal coupling at the thru and coupled port. On the other hand, the existing techniques still require a considerably large chip area, leading to a high implementation cost for system integration.

To overcome the design limitations imposed by the lumped passive components, the concept of quadrature hybrids with active inductors has been adopted, and a controllable attenuator has been realized in a pseudomorphic HEMT (pHEMT) foundry process [9]. In this paper, a miniaturized technique is presented for the design of fully integrated CMOS hybrids. By utilizing active inductors in the lumped equivalent circuit, a significant area reduction can be achieved while maintaining enhanced circuit performance at multigigahertz frequencies. In addition, the tunable inductance of the active inductors enables the control of the center frequency by the bias currents, which can be utilized for the realization of reconfigurable RF frontends in multistand wireless systems. Based on the proposed technique, a $C$-band hybrid circuit was designed and fabricated in a $0.18-\mu \mathrm{m}$ bulk CMOS process for demonstration.

This paper is organized as follows. Section II presents the realization of the CMOS active inductors, including the circuit topology, theoretical analysis, design considerations, and chip implementation. The design of the fully integrated quadrature hybrid using CMOS active inductors is described in Section III, followed by the experimental results in Section IV. Finally, conclusions are briefly outlined in Section V.

\section{CMOS ACTIVE INDUCTORS}

To alleviate the limitations imposed on the chip area and the quality $(Q)$ factors of the spiral inductors, active designs [10]-[15] were proposed to implement the required on-chip inductance. For RF applications, the regulated cascode topology is commonly used in the design of CMOS active inductors [16]. Fig. 1(a) shows the schematic of the active inductor. As the input voltage applies to the gate terminal of the common-source transistor $M_{1}$, the transconductance $g_{m 1}$ converts the voltage to a drain current charging the capacitance $C_{\mathrm{gs} 2}$ of transistor $M_{2}$. The voltage established across $C_{\mathrm{gs} 2}$ is then converted to the input current by the transconductance of $M_{2}$, emulating the current-voltage characteristics of a shunt inductance. Note that the transistor $M_{3}$ is used as the gain-boosting stage to enhance the $Q$ factor of the active inductor, while the required bias currents for $M_{1}$ and $M_{2}$ are provided by the current mirrors $M_{4}-M_{7}$. From the small-signal analysis, the nodal voltages $V_{1}$ and $V_{2}$ can be expressed as

$$
\begin{aligned}
& V_{1}=-\frac{g_{m 1}}{s C_{\mathrm{gs} 3}+g_{m 3}} V_{\mathrm{in}} \\
& V_{2}=\frac{s^{2} C_{\mathrm{gs} 2} C_{\mathrm{gs} 3}+s C_{\mathrm{gs} 2} g_{m 3}-g_{m 1} g_{m 3}}{s^{2} C_{\mathrm{gs} 2} C_{\mathrm{gs} 3}+s C_{\mathrm{gs} 2} g_{m 3}+s C_{\mathrm{gs} 3} g_{\mathrm{ds} 4}+g_{m 3} g_{\mathrm{ds} 4}} V_{\mathrm{in}}
\end{aligned}
$$




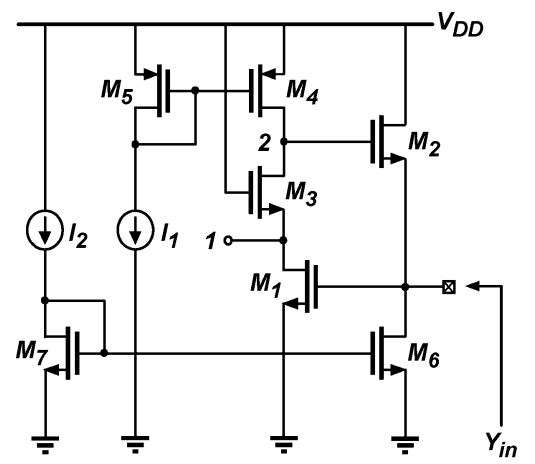

(a)

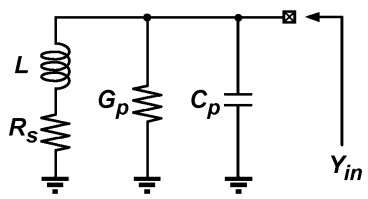

(b)

Fig. 1. (a) Schematic and (b) equivalent circuit of the regulated cascode active inductor.

and the input admittance of the active inductor is given by

$$
Y_{\mathrm{in}}=\frac{1}{Z_{\mathrm{in}}} \approx \frac{g_{m 1} g_{m 2} g_{m 3}}{s C_{\mathrm{gs} 2}\left(g_{m 3}+s C_{\mathrm{gs} 3}\right)}+\frac{g_{m 1} g_{m 3}}{g_{m 3}+s C_{\mathrm{gs} 3}}+s C_{\mathrm{gs} 1} .
$$

Assuming that the operating frequency of the active inductor is much lower than the cutoff frequency of $M_{3}$, which can be expressed as $\omega_{T 3}=g_{m 3} / C_{\mathrm{gs} 3}$, the input admittance of the active inductor can be approximated by the equivalent circuit, as shown in Fig. 1(b), and the expressions of the $L, R_{s}, G_{p}$, and $C_{p}$ are provided as

$$
\begin{aligned}
L & \approx C_{\mathrm{gs} 2} /\left(g_{m 1} g_{m 2}\right) \\
R_{s} & \approx-\omega^{2} C_{\mathrm{gs} 2} C_{\mathrm{gs} 3} /\left(g_{m 1} g_{m 2} g_{m 3}\right) \\
G_{p} & \approx g_{m 1} \\
C_{p} & \approx C_{\mathrm{gs} 1} .
\end{aligned}
$$

For an active inductor, the value of the inductance $L$ is determined by the small-signal circuit parameters of $M_{1}$ and $M_{2}$, while the $Q$ factor is strongly influenced by the values of $G_{p}$ and $R_{s}$. In the equivalent circuit, $G_{p}$ represents the shunt conductance, accounting for the loss of the active inductor. On the other hand, $R_{s}$ is a negative resistance with frequency-dependent characteristics. Based on the simplified circuit model, the resonant frequency of the active inductor is given by

$$
f_{\mathrm{RES}} \approx \frac{1}{2 \pi \sqrt{L C_{p}}}=\frac{1}{2 \pi} \sqrt{\frac{g_{m 1}}{C_{\mathrm{gs} 1}}} \cdot \sqrt{\frac{g_{m 2}}{C_{\mathrm{gs} 2}}} \approx \frac{1}{2 \pi} \sqrt{\omega_{T 1} \omega_{T 2}}
$$

Note that $\omega_{T 1}$ and $\omega_{T 2}$ are the cutoff frequencies of $M_{1}$ and $M_{2}$, respectively, which impose a fundamental limitation on the operating frequencies of the active inductors. Typically, the active inductors are operated at frequencies much lower than the resonant frequency to ensure the desirable circuit characteristics.

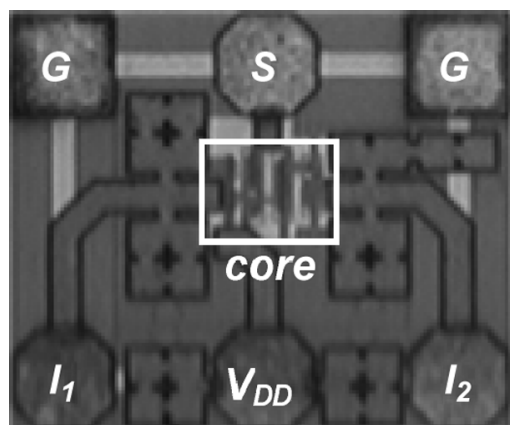

Fig. 2. Die photograph of the fabricated active inductor.

TABLE I

CIRCUIT PARAMETERS OF THE FABRICATED ACTIVE INDUCTOR

\begin{tabular}{|c|c|}
\hline Transistors & Size $(\mu \mathrm{m} / \mu \mathrm{m})$ \\
\hline$M_{1}$ & $100 / 0.18$ \\
\hline$M_{2}$ & $60 / 0.18$ \\
\hline$M_{3}$ & $10 / 0.18$ \\
\hline$M_{4}$ & $40 / 0.18$ \\
\hline$M_{5}$ & $40 / 0.18$ \\
\hline$M_{6}$ & $10 / 0.18$ \\
\hline$M_{7}$ & $20 / 0.18$ \\
\hline
\end{tabular}

To optimize the inductor performance, the transistor parameters can be properly chosen such that $R_{s}$ compensates for the loss from $G_{p}$ at the frequency of interest

$\operatorname{Re}\left[\frac{1}{R_{s}+j \omega L}\right]+g_{m 1}=\frac{-C_{\mathrm{gs} 2} C_{\mathrm{gs} 3} g_{m 1} g_{m 2} g_{m 3}}{C_{\mathrm{gs} 2}^{2} g_{m 3}^{2}+\omega^{2} C_{\mathrm{gs} 2}^{2} C_{\mathrm{gs} 3}^{2}}+g_{m 1}=0$.

As a result, a peak $Q$ factor can be achieved at

$$
f_{Q_{\max }} \approx \frac{1}{2 \pi} \sqrt{\omega_{T 3}\left(\omega_{T 2}-\omega_{T 3}\right)}
$$

In order to evaluate the broadband characteristics of the active inductors, the quality factor is defined as the ratio of the imaginary part to the real part of the input impedance, which can be approximated by

$$
Q \approx \frac{\omega^{2} C_{\mathrm{gs} 2} g_{m 1} g_{m 2} g_{m 3}^{2}}{g_{m 1} g_{m 2} g_{m 3}^{2} g_{\mathrm{ds} 4}+\omega^{2} C_{\mathrm{gs} 2} g_{m 1} g_{m 3}\left(C_{\mathrm{gs} 2} g_{m 3}-C_{\mathrm{gs} 3} g_{m 2}\right)} .
$$

Other than the reduced chip area and the enhanced $Q$ factor, another advantage of using active inductors is the tuning capability of the inductance values. From (4), the value of $L$ is governed by the transconductances $g_{m 1}$ and $g_{m 2}$. Hence, the equivalent inductance can be adjusted by the bias currents $I_{1}$ and $I_{2}$, resulting in another degree of freedom for the circuit operation.

In (4), the value of the inductance $L$ is determined by the transconductance $g_{m 1}$ and $g_{m 2}$, which are considered constant under small-signal approximation. However, it is not very accurate for the active inductors operating in the large-signal 


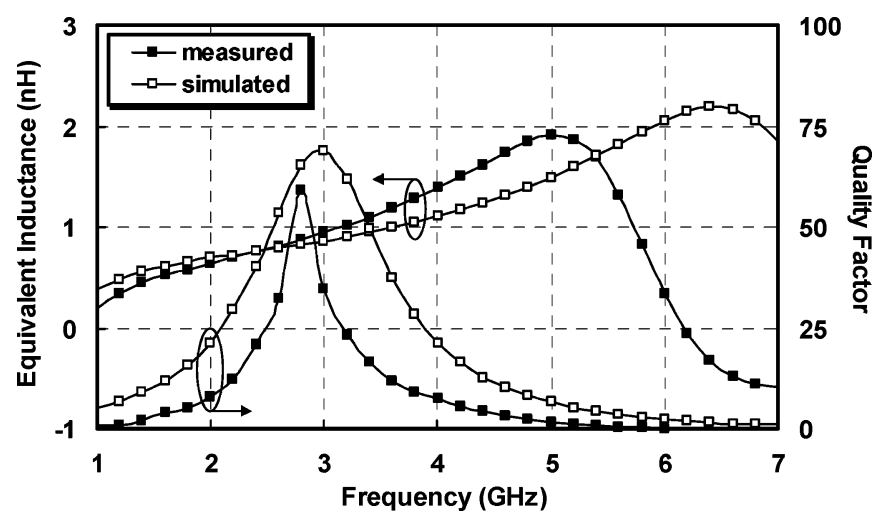

Fig. 3. Simulated and measured small-signal characteristics of the active inductor with $I_{1}=1.5 \mathrm{~mA}$ and $I_{2}=1.6 \mathrm{~mA}$.

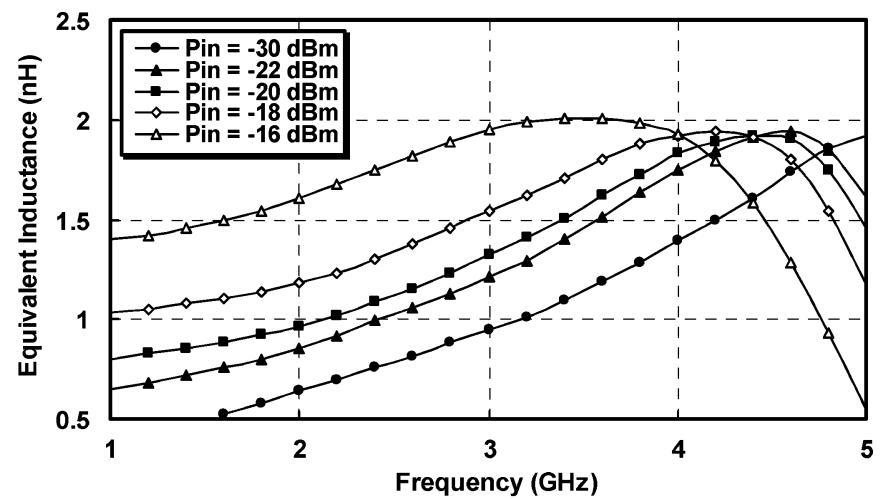

(a)

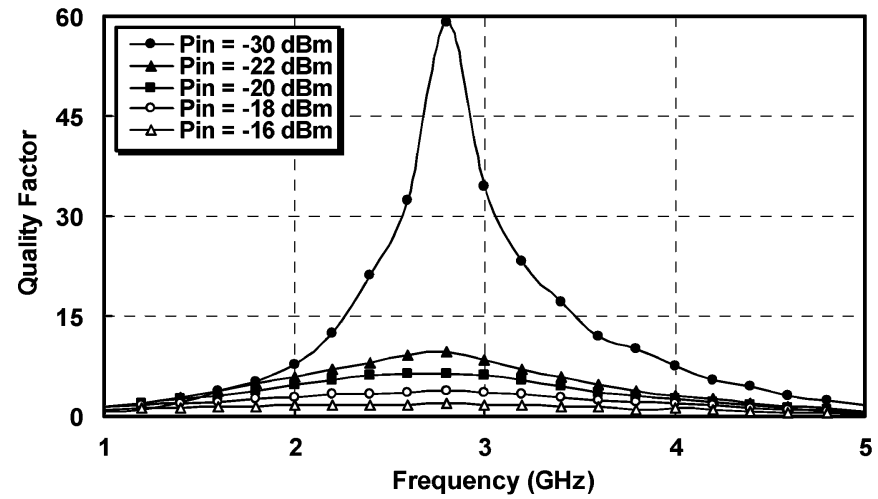

(b)

Fig. 4. Measured: (a) equivalent inductance and (b) $Q$ factor of the regulated cascode active design with various incident power levels.

mode. As the amplitude of the signal power increases, the excess voltage swing leads to a decrease in the transconductance of the transistors. The inductance $L$ thus deviates from its small-signal value due to the nonlinear characteristics, resulting in undesirable signal distortion. Therefore, the impact of the linearity issues on the circuit performance should be carefully examined when the active inductors are used to replace the spiral inductors in MMIC designs. For a CMOS active inductor using the regulated cascode topology, the deviation in the transconductance due to large-signal operations can be effectively minimized by increasing the overdrive voltage and

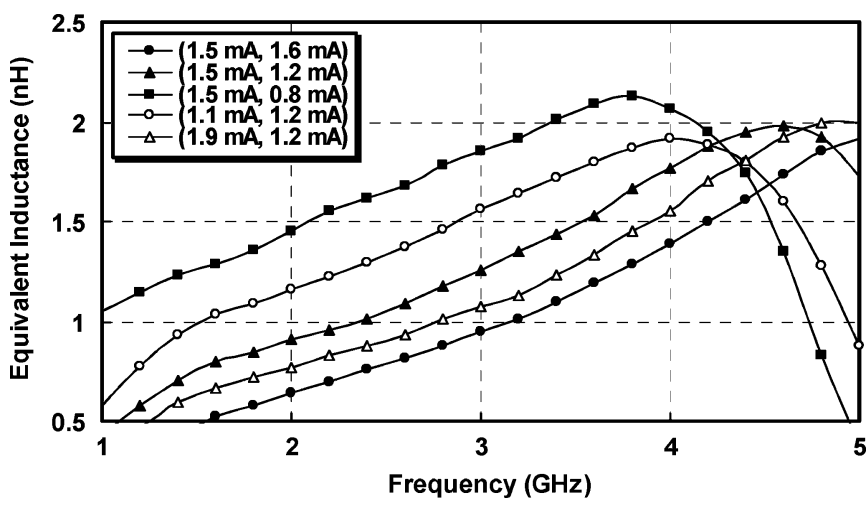

Fig. 5. Measured equivalent inductance at various bias currents $\left(I_{1}, I_{2}\right)$.

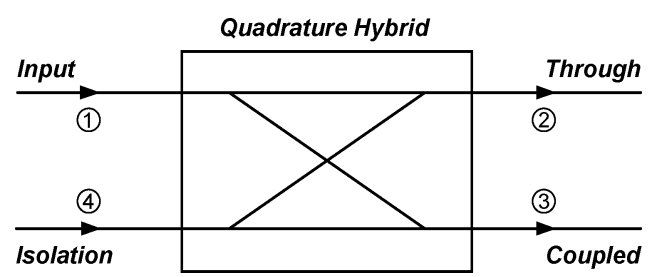

Fig. 6. Port assignment of the quadrature hybrid.

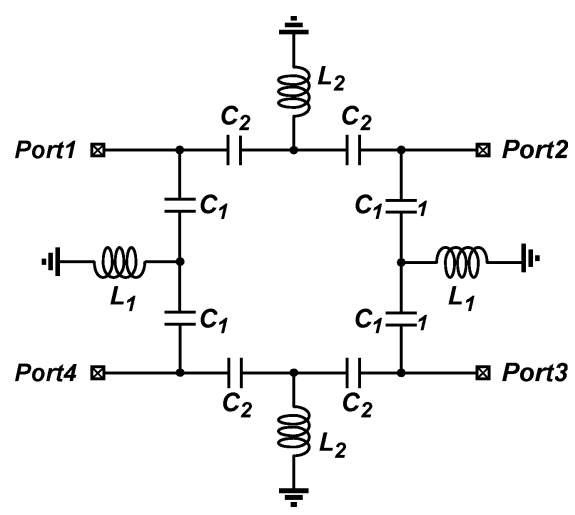

Fig. 7. Equivalent circuit model of a lumped hybrid.

by reducing the transistor size at the expense of an elevated supply voltage.

In order to characterize the small- and large-signal performance of the active inductors for RF applications, a standard $0.18-\mu \mathrm{m}$ CMOS process was employed for the circuit implementation. The microphotograph of the fabricated circuit is shown in Fig. 2, while the design parameters are tabulated in Table I. With $I_{1}=1.5 \mathrm{~mA}$ and $I_{2}=1.6 \mathrm{~mA}$, the small-signal characteristics of the active inductor are illustrated in Fig. 3. Note that the equivalent inductance $L_{\mathrm{eq}}$ is defined here as

$$
L_{\mathrm{eq}}=\frac{\operatorname{Im}\left(Z_{\mathrm{in}}\right)}{\omega} \text {. }
$$

Based on the experimental results, the fabricated circuit exhibits an equivalent inductance of $0.88 \mathrm{nH}$ with a maximum $Q$ factor of 59 at $2.8 \mathrm{GHz}$. In such a specified bias condition, the large-signal behavior of the active inductor is also characterized by measuring the input reflection coefficient with various incident signal power. The impacts of the incident power level $\left(P_{\text {in }}\right)$ on the equivalent inductance and $Q$ factor are shown in 


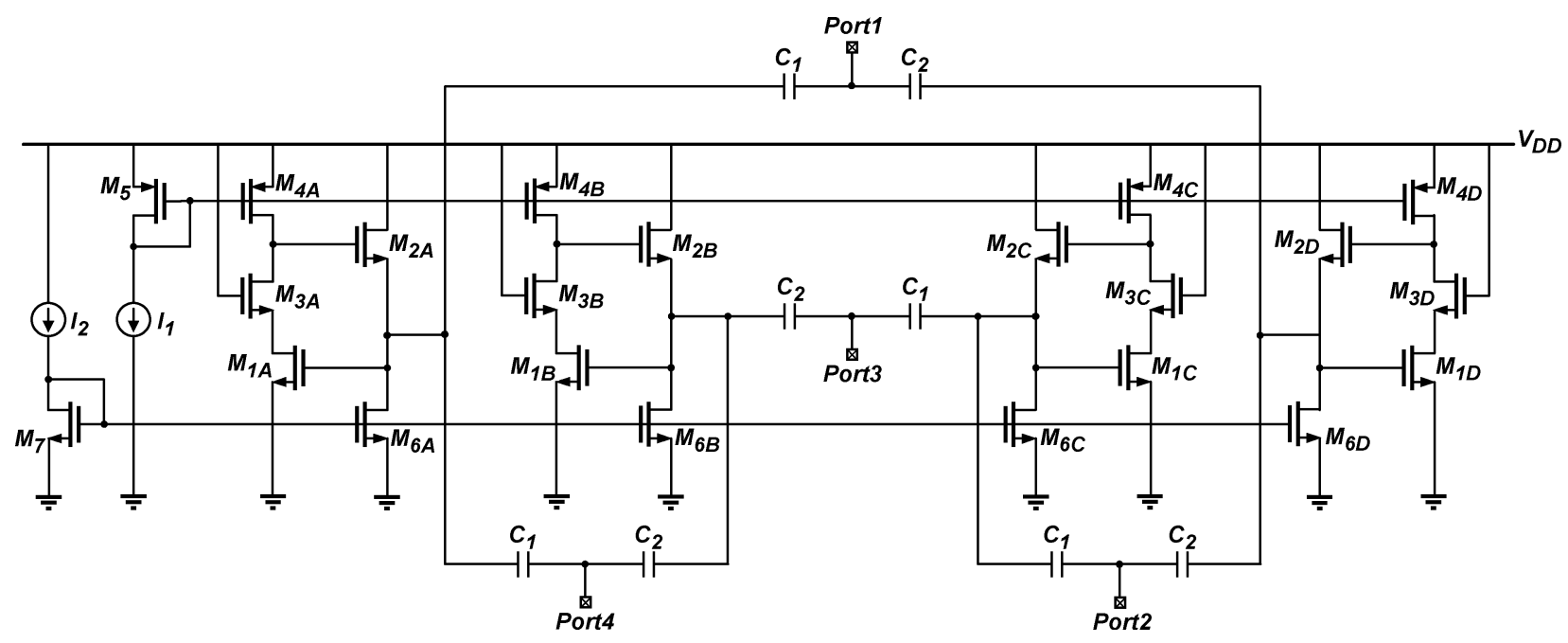

Fig. 8. Complete circuit schematic of the fully integrated quadrature hybrid.

Fig. 4(a) and (b), respectively. It is noted that the equivalent inductance increases with the signal power level due to the decreasing transconductances of the MOS devices. Since the transistors may operate in the triode regions at large-signal operations, the $Q$ factor tends to degrade at higher input power level. Another important feature of the active inductor is the tunability. By varying the bias currents of $I_{1}$ and $I_{2}$, the measured equivalent inductance as a function of frequency is illustrated in Fig. 5, indicating a wide inductance tuning range at multigigahertz frequencies.

\section{DESIGn OF MMIC QUADRATURE HYBRID}

The port assignment and functionality of a quadrature hybrid are shown in Fig. 6. For a planar process technology, the circuit is conventionally implemented by branch-line couplers due to its simplicity [17], [18]. In consideration of the prohibitively large chip area, lumped-element topologies have been proposed to realize the hybrid circuits for applications below $10 \mathrm{GHz}$ [4]-[7]. With reactive elements including shunt inductors and series capacitors, the equivalent circuit model of a lumped hybrid at its center frequency $f_{0}$ is shown in Fig. 7, where the values of the components are given as

$$
\begin{aligned}
& C_{2}=\sqrt{2} \cdot C_{1}=\frac{\sqrt{2}}{2 \pi f_{0} Z_{0}} \approx \frac{1}{222 f_{0}} \\
& L_{2}=\frac{1}{\sqrt{2}} \cdot L_{1}=\frac{Z_{0}}{2 \sqrt{2} \pi f_{0}} \approx \frac{5.6}{f_{0}} .
\end{aligned}
$$

To demonstrate the potential of monolithic system integration, a 1P6M 0.18- $\mu \mathrm{m}$ CMOS process provided by a commercial foundry is used to fabricate the proposed quadrature hybrid. With a device layout optimized for the RF performance, the MOSFETs exhibit a maximum oscillation frequency $f_{\max }$ up to $60 \mathrm{GHz}$. As for the backend process technology, a top $\mathrm{AlCu}$ metallization layer of $2-\mu \mathrm{m}$ thickness is available for the interconnection, while a metal-insulator-metal (MIM) structure with a capacitance density of $1 \mathrm{fF} / \mu \mathrm{m}^{2}$ is also provided. With a center frequency of $4 \mathrm{GHz}$, the required inductances and capacitances in this design can be calculated from (13) and (14).
TABLE II

Circuit PARAMETERS OF THE QUADRATURE HYBRID

\begin{tabular}{|c|c|c|}
\hline Device & Unit & Designed Value \\
\hline$M_{I A}, M_{I C}$ & $\mu \mathrm{m} / \mu \mathrm{m}$ & $130 / 0.18$ \\
\hline$M_{1 B}, M_{I D}$ & $\mu \mathrm{m} / \mu \mathrm{m}$ & $100 / 0.18$ \\
\hline$M_{2 A}, M_{2 C}$ & $\mu \mathrm{m} / \mu \mathrm{m}$ & $50 / 0.18$ \\
\hline$M_{2 B}, M_{2 D}$ & $\mu \mathrm{m} / \mu \mathrm{m}$ & $60 / 0.18$ \\
\hline$M_{3 A}, M_{3 B}, M_{3 C}, M_{3 D}$ & $\mu \mathrm{m} / \mu \mathrm{m}$ & $10 / 0.18$ \\
\hline$M_{4 A}, M_{4 B}, M_{4 C}, M_{4 D}$ & $\mu \mathrm{m} / \mu \mathrm{m}$ & $40 / 0.18$ \\
\hline$M_{6 A}, M_{6 C}$ & $\mu \mathrm{m} / \mu \mathrm{m}$ & $20 / 0.18$ \\
\hline$M_{6 B}, M_{6 D}$ & $\mu \mathrm{m} / \mu \mathrm{m}$ & $10 / 0.18$ \\
\hline$M_{5}$ & $\mu \mathrm{m} / \mu \mathrm{m}$ & $40 / 0.18$ \\
\hline$M_{7}$ & $\mu \mathrm{m} / \mu \mathrm{m}$ & $20 / 0.18$ \\
\hline$C_{I}$ & $\mathrm{pF}$ & 0.80 \\
\hline$C_{2}$ & $\mathrm{pF}$ & 1.06 \\
\hline
\end{tabular}

The obtained $L_{1}$ and $L_{2}$ are 2.0 and $1.4 \mathrm{nH}$, respectively, while $C_{1}$ is $0.8 \mathrm{pF}$ and $C_{2}$ is $1.1 \mathrm{pF}$. For the circuit implementation, the shunt inductances in the lumped circuit model are realized by the regulated cascode active inductors, and MIM capacitors provided by the standard CMOS process are used for the capacitive elements. The complete circuit schematic of the fully integrated quadrature hybrid is depicted in Fig. 8, where the design parameters are tabulated in Table II.

Due to the use of active inductors, no distributed elements or spiral inductors are required. A significant reduction in chip area can be achieved. In addition, the resulting high- $Q$ factors of the active inductors also enhance the hybrid performance compared with the lumped-element topology. Another important feature of the proposed technique is the reconfigurability in the operating frequency bands. As indicated in (4), the inductance of an active inductor is strongly influenced by the bias currents of the MOSFETs. Therefore, the center frequency of the quadrature hybrid can be effectively tuned by the bias currents to compensate for the process variation or to realize a multiband RF functionality. 


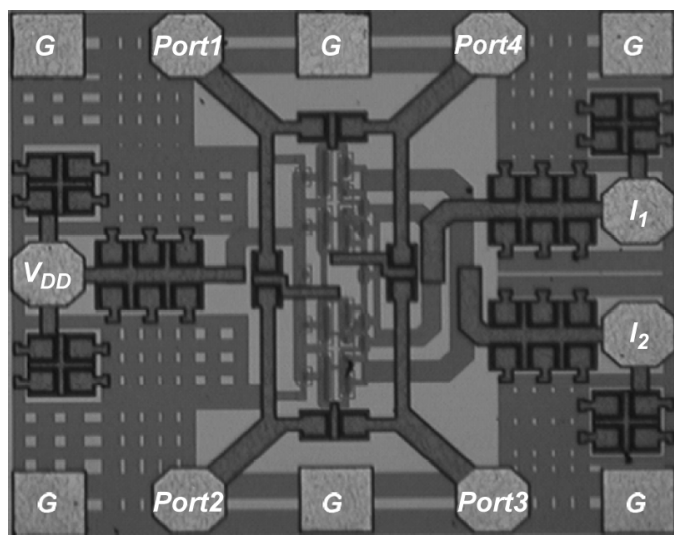

Fig. 9. Die photograph of the fabricated quadrature hybrid.

\section{EXPERIMENTAL RESULTS}

Fig. 9 shows a microphotograph of the fabricated circuit. In this design, the total chip area including the pads is $0.72 \times$ $0.64 \mathrm{~mm}^{2}$, where the active circuit area occupies only $0.4 \times$ $0.2 \mathrm{~mm}^{2}$. Compared with conventional branch-line hybrids at the same frequency band, the chip area is reduced by several orders of magnitude.

To characterize the $S$-parameters of the quadrature hybrid, on-wafer probing was performed by using a multiport vector network analyzer. No on-chip terminations are required and the impedance mismatch during the measurement can be minimized for better accuracy. When the circuit is biased for operation at a center frequency of $4.2 \mathrm{GHz}$, the bias currents $I_{1}$ and $I_{2}$ are both $1.5 \mathrm{~mA}$. The hybrid circuit consumes a dc power of $20.4 \mathrm{~mW}$ from a $1.8-\mathrm{V}$ supply voltage. The measured $S$-parameters and output phase of the fabricated circuit are shown in Fig. 10(a) and (b), respectively. The insertion losses at the thu and the coupled port are 3.3 and $3.2 \mathrm{~dB}$, respectively, with a phase difference of $91.2^{\circ}$ at the center frequency, while the return loss and port isolation are 28 and $15 \mathrm{~dB}$, respectively. The bandwidth, which is defined as the frequency range, where $\left|S_{11}\right|<-15 \mathrm{~dB}$, is $9.5 \%$. Within this bandwidth, the insertion loss maintains lower than $4.0 \mathrm{~dB}$ and the phase error is less than $3.5^{\circ}$.

Due to the use of active devices in the circuit design, the nonlinear behavior of the quadrature hybrid is characterized by its input-referred 1-dB compression point $\left(P_{\mathrm{in}-1 \mathrm{~dB}}\right)$ between the input and output port. With ports 3 and 4 terminated, Fig. 11 shows the measured output power and the associated $\left|S_{21}\right|$ at the thu port versus the input power level. From the measurement results, $P_{\mathrm{in}-1 \mathrm{~dB}}$ is $-16 \mathrm{dBm}$, and $\left|S_{21}\right|$ tends to decrease as the input power level increases. For a conventional quadrature hybrid with passive components, the noise figure is simply evaluated by the insertion loss from the input to the output port. As for the active hybrid circuit, however, excess noise sources from the MOSFETs have to be taken into account. It is more complicated to define and to extract the noise figure experimentally for the four-port network. In this study, a simplified measurement setup has been adopted to evaluate the noise figure. By treating the quadrature hybrid as a two-port network with $50-\Omega$ terminations for the isolation and the coupled port, the measured noise figure from the input to the through port is $17 \mathrm{~dB}$ at the center

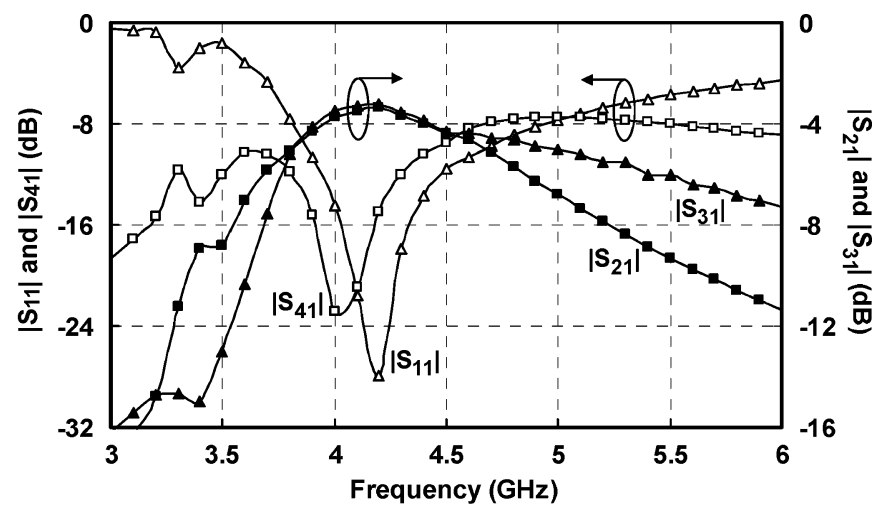

(a)

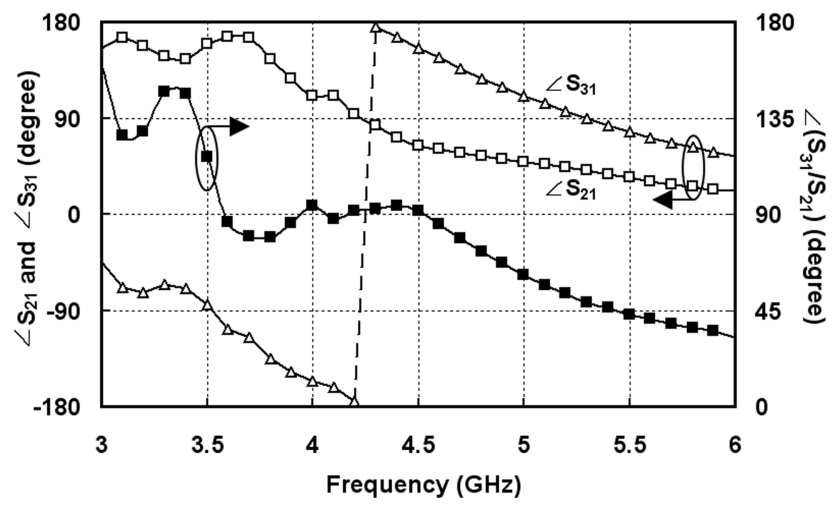

(b)

Fig. 10. Measured: (a) $S$-parameters and (b) output phase of the fabricated quadrature hybrid.

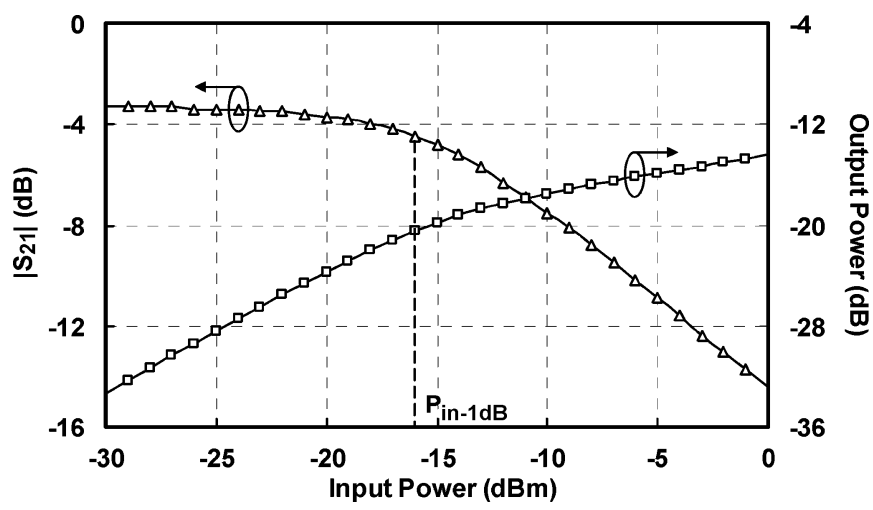

Fig. 11. Measured large-signal behavior of the quadrature hybrid.

frequency of $4 \mathrm{GHz}$. It is noted that the measurement accuracy may be influenced by the port reflections due to impedance mismatch and by the thermal noise from the resistive loads. Therefore, it tends to overestimate and can only be considered an approximated specification for design reference.

One of the unique features for the proposed hybrid circuit is the reconfigurability provided by the tunable active inductors. By adjusting the equivalent inductance through the bias currents $I_{1}$ and $I_{2}$, the center frequency can be effectively tuned over a wide frequency range. For the operation at the lower frequency band, the bias current $I_{1}$ and $I_{2}$ are set to 1.1 and 


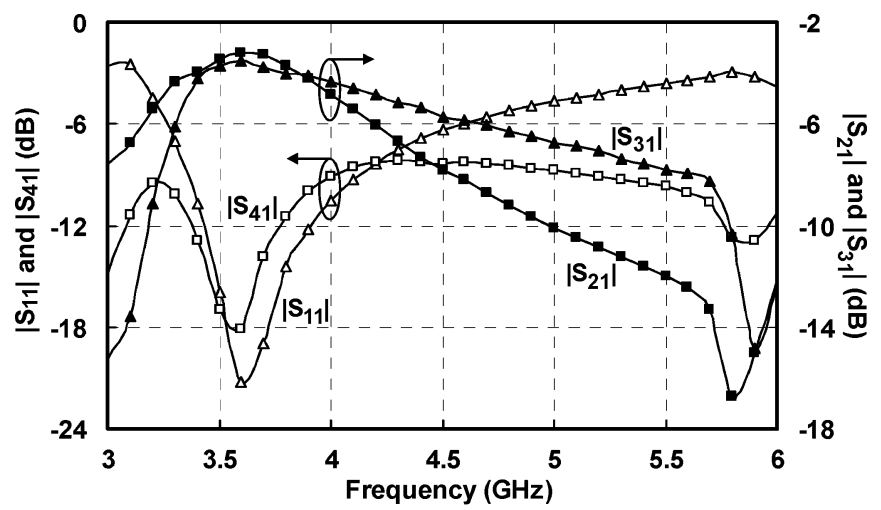

(a)

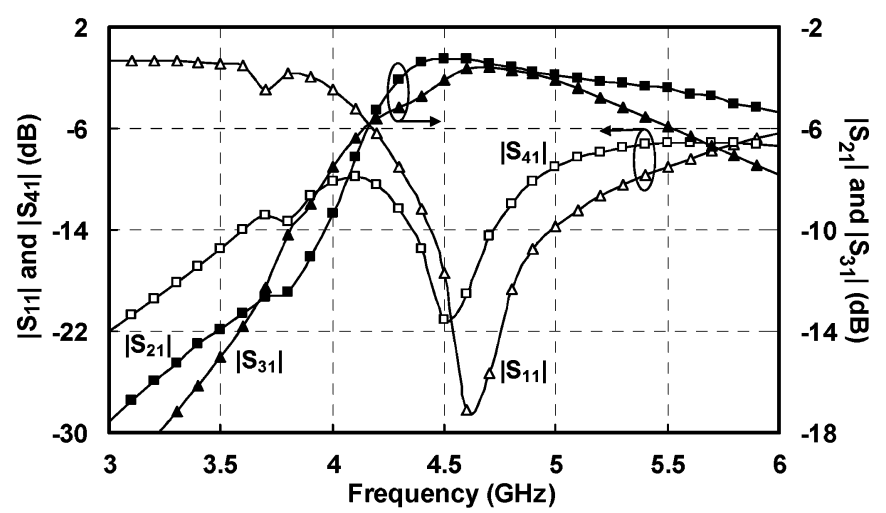

(b)

Fig. 12. Measured $S$-parameters of the quadrature hybrid at the: (a) lower and (b) upper operating frequency bands.

TABLE III

PERFORMANCE SUMMARY

\begin{tabular}{|c|c|c|c|}
\hline \multicolumn{4}{|c|}{ Quadrature Hybrid Using Active Inductors } \\
\hline Technology & \multicolumn{3}{|c|}{$0.18-\mu \mathrm{m}$ CMOS } \\
\hline Chip Size & \multicolumn{4}{|c|}{$0.72 \times 0.64 \mathrm{~mm}^{2}$} \\
\hline Active Area & \multicolumn{4}{|c|}{$0.4 \times 0.2 \mathrm{~mm}^{2}$} \\
\hline Center Frequency $(\mathrm{GHz})$ & 3.6 & 4.2 & 4.7 \\
\hline$\left|\mathrm{S}_{21}\right|(\mathrm{dB})$ & -3.2 & -3.3 & -3.4 \\
\hline$\left|\mathrm{S}_{31}\right|(\mathrm{dB})$ & -3.5 & -3.2 & -3.5 \\
\hline Return Loss $(\mathrm{dB})$ & 21 & 28 & 25 \\
\hline Isolation $(\mathrm{dB})$ & 18 & 15 & 14 \\
\hline Output Phase Error $\left(^{\circ}\right)$ & 2 & 1.2 & 3.5 \\
\hline DC Power $(\mathrm{mW})$ & 17.6 & 20.4 & 24.6 \\
\hline
\end{tabular}

$1.4 \mathrm{~mA}$, respectively. The resulting inductances of $L_{1}$ and $L_{2}$ are approximately 2.4 and $1.8 \mathrm{nH}$, respectively, leading to a shift of the center frequency from its midband value of 4.2 to $3.6 \mathrm{GHz}$. On the other hand, with $I_{1}$ of $1.9 \mathrm{~mA}$ and $I_{2}$ of $3.0 \mathrm{~mA}$, the equivalent inductances of $L_{1}$ and $L_{2}$ are 1.4 and $1.1 \mathrm{nH}$, respectively, effectively moving the center frequency of the hybrid to $4.7 \mathrm{GHz}$. The measured $S$-parameters for the lower and the upper operating frequency bands are illustrated in Fig. 12(a) and (b), respectively. Table III summaries the performance of the fabricated quadrature hybrid at various operating frequency bands. According to the measurement results, the proposed circuit is suitable for reconfigurable RF applications with a center frequency from 3.6 to $4.7 \mathrm{GHz}$. Within the entire frequency range of operation, the measured $\left|S_{21}\right|$ and $\left|S_{31}\right|$ of the quadrature hybrid are higher than $-4 \mathrm{~dB}$ with an output phase error less than $4^{\circ}$, while the return loss and isolation are generally better than 20 and $10 \mathrm{~dB}$, respectively.

\section{CONCLUSION}

A tunable active inductor has been implemented in a standard $0.18-\mu \mathrm{m}$ CMOS process. With the analysis and characterization of the fabricated circuit, the small-signal performance and nonlinear behavior of the CMOS active inductors have been presented for MMIC designs at multigigahertz frequencies. Based on the development of the active inductor, a compact quadrature hybrid has been demonstrated at a center frequency of $4.2 \mathrm{GHz}$. Due to the absence of distributed components and spiral inductors, the fully integrated hybrid only occupies an active chip area of $0.4 \times 0.2 \mathrm{~mm}^{2}$. With the high- $Q$ factors and tunable inductance provided by the active inductors, the quadrature hybrid exhibits enhanced performance in terms of insertion loss, impedance matching, and port isolation while maintaining a frequency tuning range of $1.1 \mathrm{GHz}$.

\section{ACKNOWLEDGMENT}

The authors would like to thank the National Chip Implementation Center (CIC), Hsinchu, Taiwan, R.O.C., for chip fabrication and National Nano Device Laboratories (NDL), Hsinchu, Taiwan, R.O.C., for chip measurement. The authors are also grateful to Y.-C. Tarn, National Taiwan University, Taipei, Taiwan, R.O.C., for measurement support.

\section{REFERENCES}

[1] T. Tokumitsu et al., "Multilayer MMIC using a $3 \mu \mathrm{m} \times 3$-layer dielectric film structure," in IEEE MTT-S Int. Microw. Symp. Dig., May 1990, vol. 2, pp. 831-834.

[2] I. Toyoda et al., "Multilayer MMIC branch-line coupler and broad-side coupler," in IEEE Microw. Millimeter-Wave Monolithic Circuits Symp., Jun. 1992, pp. 79-82.

[3] C. Y. Ng, M. Chongcheawchamnan, and I. D. Robertson, "Lump-distributed hybrids in 3D-MMIC technology," Proc. Inst. Elect. Eng.-Microw., Antennas, Propag., vol. 151, no. 4, pp. 370-374, Aug. 2004.

[4] Y. C. Chiang and C. Y. Chen, "Design of lumped element quadrature hybrid," Electron. Lett., vol. 34, no. 5, pp. 465-467, Mar. 1998.

[5] L.-H. Lu et al., "Design and implementation of micromachined lumped quadrature $\left(90^{\circ}\right)$ hybrids," in IEEE MTT-S Int. Microw. Symp. Dig., May 2001, vol. 2, pp. 1285-1288.

[6] R. C. Frye, S. Kapur, and R. C. Melville, "A 2-GHz quadrature hybrid implemented in CMOS technology," IEEE J. Solid-State Circuits, vol. 38, no. 3, pp. 550-555, Mar. 2003.

[7] W.-S. Tung, H.-H. Wu, and Y.-C. Chiang, "Design of microwave wideband quadrature hybrid using planar transformer coupling method,' IEEE Trans. Microw. Theory Tech., vol. 51, no. 7, pp. 1852-1856, Jul. 2003.

[8] T. Hirota, A. Minakawa, and M. Muraguchi, "Reduced-size branchline and rat-race hybrids for uniplanar MMIC's," IEEE Trans. Microw. Theory Tech., vol. 38, no. 3, pp. 270-275, Mar. 1990.

[9] G. Avitabile et al., "A $5.8 \mathrm{GHz}$ ISM band active $90^{\circ}$ hybrid and variable attenuator," Microw. Opt. Technol. Lett., vol. 36, no. 5, pp. 325-327, Feb. 2003.

[10] L.-H. Lu, H.-H. Hsieh, and Y.-T. Liao, "A wide tuning-range CMOS VCO with a differential tunable active inductor," IEEE Trans. Microw. Theory Tech., vol. 54, no. 9, pp. 3462-3468, Sep. 2006.

[11] L.-H. Lu, Y.-T. Liao, and C.-R. Wu, "A miniaturized Wilkinson power divider with CMOS active inductors," IEEE Microw. Wireless Compon. Lett., vol. 15, no. 11, pp. 775-777, Nov. 2005. 
[12] L.-H. Lu and Y.-T. Liao, "A 4-GHz phase shifter MMIC in 0.18- $\mu \mathrm{m}$ CMOS," IEEE Microw. Wireless Compon. Lett., vol. 15, no. 10, pp. 694-696, Oct. 2005.

[13] R. Mukhopadhyay et al., "Reconfigurable RFICs in Si-based technologies for a compact intelligent RF front-end," IEEE Trans. Microw. Theory Tech., vol. 53, no. 1, pp. 81-93, Jan. 2005.

[14] Y. Wu et al., "RF bandpass filter design based on CMOS active inductors," IEEE Circuits Syst. II, Analog Digit. Signal Process., vol. 50, no. 12, pp. 942-949, Dec. 2003.

[15] C.-C. Hsiao et al., "Improved quality-factor of 0.18- $\mu \mathrm{m}$ CMOS active inductor by a feedback resistance design," IEEE Microw. Wireless Compon. Lett., vol. 12, no. 12, pp. 467-469, Dec. 2002.

[16] A. Thanachayanont and A. Payne, "VHF CMOS integrated active inductor," Electron. Lett., vol. 32, no. 11, pp. 999-1000, May 1996.

[17] C. Collado, A. Grau, and F. D. Flaviis, "Dual-band planar quadrature hybrid with enhanced bandwidth response," IEEE Trans. Microw. Theory Tech., vol. 54, no. 1, pp. 180-188, Jan. 2006.

[18] D. M. Pozar, Microwave Engineering, 3rd ed. New York: Wiley, 2005.

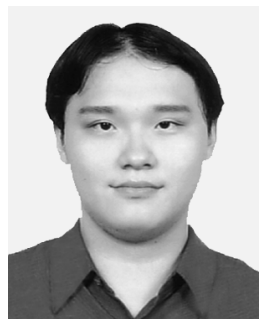

Hsieh-Hung Hsieh (S'05) was born in Taipei, Taiwan, R.O.C., in 1981. He received the B.S. degree in electrical engineering from National Taiwan University, Taipei, Taiwan, R.O.C., in 2004, and is currently working toward the Ph.D. degree in electronic engineering at National Taiwan University.

His research interests include the development of low-voltage and low-power RF integrated circuits, multiband wireless systems, RF testing, and monolithic microwave integrated circuit (MMIC) designs.

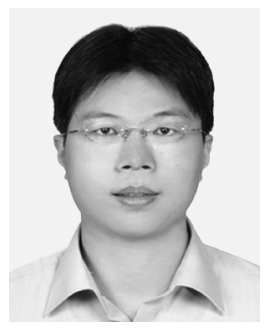

Yu-Te Liao (S’05) was born in Taichung, Taiwan, R.O.C., in 1981. He received the B.S. degree in electrical engineering from National Cheng Kung University, Tainan, Taiwan, R.O.C., in 2003, the M.S. degree in electronics engineering from National Taiwan University, Taipei, Taiwan, R.O.C., in 2005, and is currently working toward the Ph.D. degree at the University of Washington, Seattle.

His research interests include RF integrated circuits, wideband frequency synthesizers, and wireless sensor network interface designs.

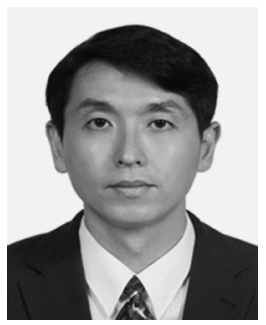

Liang-Hung Lu (M'02) was born in Taipei, Taiwan, R.O.C., in 1968. He received the B.S. and M.S. degrees in electronics engineering from National Chiao-Tung University, Hsinchu, Taiwan, R.O.C., in 1991 and 1993, respectively, and the Ph.D. degree in electrical engineering from The University of Michigan at Ann Arbor, in 2001.

During his graduate study, he was involved in $\mathrm{SiGe}$ HBT technology and MMIC designs. From 2001 to 2002 , he was with IBM, where he was involved with low-power and RF integrated circuits for silicon-oninsulator (SOI) technology. In August 2002, he joined the faculty of the Graduate Institute of Electronics Engineering and the Department of Electrical Engineering, National Taiwan University, Taipei, Taiwan, R.O.C., where he is currently an Associate Professor. His research interests include CMOS/BiCMOS $\mathrm{RF}$ and mixed-signal integrated-circuit designs. 